\title{
Senno del poi o senno di por?
}

\section{Massimo Fanfani}

PUBBLICATO: 22 GENNAIO 2021

\section{Quesito:}

In questi ultimi tempi diverse persone attente e sensibili ai fatti di lingua si sono rivolte al servizio di Consulenza linguistica dell'Accademia della Crusca per sapere se sia corretto dire col senno del poi, o non piuttosto col senno di poi.

\section{Senno del poi o senno di poi?}

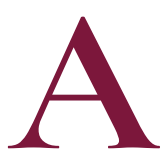

i più la questione potrà apparire di scarso rilievo e del tutto trascurabile: in fondo si tratta di una scelta a prima vista piuttosto semplice fra un avverbio (di poi) e un avverbio sostantivato (del poi), apparentemente senza grosse conseguenze sul senso complessivo e il tono dell'espressione: col senno di poi 'col senno che si manifesta successivamente, a posteriori' col senno del poi 'col senno del tempo venturo'.

Certuni la riterranno addirittura una questione oziosa: la soluzione "giusta" non la si legge già in ogni vocabolario? Eppure la domanda è interessante, perché, confermando che continua a persistere nell'uso contemporaneo un'irriducibile oscillazione fra le due varianti, rivela la forte tendenza, tutta moderna, a voler superare tale incertezza e a puntare su una forma univoca, fino al punto da appellarsi alla Crusca.

Per cominciare va detto che l'espressione in questione, come avviene anche in altri casi, è nata dalla costola di un proverbio che un tempo era abbastanza noto: Del senno di poi ne son piene le fosse. Lo si trova attestato nella seconda metà del Cinquecento in diversi scrittori toscani (Agnolo Firenzuola, Anton Francesco Grazzini, Giovanni Maria Cecchi, Ludovico Guicciardini) e, naturalmente, nello sterminato repertorio di proverbi che Francesco Serdonati compilo fra la fine del Cinquecento e l'inizio del Seicento (vedi l'Appendice). Nell'Ottocento sarà ripreso, tra gli altri, anche da Manzoni nel XXIV capitolo dei Promessi sposi, cosicché dopo di allora, almeno fin quando si è letto il romanzo per intero, poté godere di una più larga circolazione.

Nelle varie attestazioni scritte che ne abbiamo il proverbio si presenta talvolta con delle piccole varianti nella sua seconda parte: omissione del ne, piene sostituito con ripiene, ecc. Ma nella prima parte esso resta immutato: del senno di poi. In tal forma è registrato in tutti i vocabolari, che in ciò seguono i nostri maggiori scrittori.

Tuttavia, nonostante i vocabolari e l'esempio manzoniano, dalla fine del secolo XIX il proverbio comincia a diffondersi in una variante che tocca la prima parte: del senno del poi. Il primo a usarla, secondo quanto ho potuto reperire, è l'eterodosso scrittore livornese Francesco Domenico Guerrazzi in una delle opere della sua maturità, la storia del bandito romano dei tempi di Sisto V, Paolo Pelliccioni (Milano, Guigoni, I864, p. Ir6): "Del senno del poi ne vanno piene le fosse".

Sebbene le prime attestazioni del proverbio, come si è visto, siano cinquecentesche, esso è sicuramente d'origine più remota: anche Manzoni lo giudica "antico". Nella tradizione classica si ritrovano adagi e proverbi esprimenti concetti analoghi, relativi all'insipienza dei mortali che non 
riescono a valutare un fatto o un comportamento se non a posteriori, quando è ormai troppo tardi e non si può rimediare. Cicerone, ad esempio, tramanda la massima: "Posteriores enim cogitationes, sapentiores solent esse". Si veda a questo proposito l'ottimo Dizionario delle sentenze latine e greche di Renzo Tosi (Milano, Rizzoli, I99I, n. I586; nella terza edizione del 20I8, n. 2086).

Ma nel nostro proverbio, come in altri proverbi consimili (Di buone intenzioni è lastricato l'Inferno; Il giudizio vien tre giorni dopo la morte; Il pentirsi da sezzo poco vale; Non aspettate il passo estremo, che molti n'ha ingannati il benfaremo; Le orazioni al capezzale non fan bene né fan male; ecc.), si avverte un'eco che nelle massime degli antichi non c'era: l'eco della concezione cristiana della vita. Le fosse, infatti, non son quelle dei campi, bensì del camposanto, a indicare quell'aldilà nel quale i conti, per chi crede nella vita eterna, vengon definitivamente saldati per ciascuno, tanto che solo dopo la morte si conosce per davvero come si sarebbe dovuto vivere. E lo conoscono i morti e non i viventi: come chi è passato all'altra riva non può cambiare alcunché della sua vita ma deve sperar solo nella redenzione, così chi ancora erra nella valle delle lacrime non si preoccupa di ciò che lo attende quasi fosse cieco davanti al suo destino. Che fra le due rive la comunicazione sia assai difficoltosa, se non impossibile, lo ricorda Abramo al ricco Epulone nella parabola evangelica (Luca, I6, I9-3I): "neque si quis ex mortuis resurrexerit, credent".

Di conseguenza, sebbene il proverbio si trovi attestato in scrittori comico-realistici del Rinascimento, è probabile che esso sia nato in ambito diverso e in un'epoca precedente: forse dal pulpito di qualche predicatore che intendeva serrare in una frase un insegnamento morale di fondamentale importanza per ogni credente. Che il proverbio fosse importante lo si capisce anche dal gran numero di "proverbisatelliti" che vi si accostano o ne sono generati. Oltre a quelli cui si è accennato poco sopra, si possono rammentare: Il cataletto porta all'intelletto; Il giudizio ci arriva sempre tardi; Il senno vien con gli anni; Vano è il piangere sul latte versato; A nave persa, tutti capitani; ecc.

Il modo di dire col (del) senno del poi, come si è notato, deriva dal proverbio e ne raccoglie l'essenza. Tale fenomeno derivativo (da proverbio a modo di dire) non è insolito e poggia sul fatto che talora $\mathrm{i}$ proverbi, pur rimanendo tali, si presentano per brevità in forma scorciata: a buon intenditore, a caval donato, campa cavallo!, carta canta, gallina vecchia, occhio non vede, perseverare é diabolico, tra il dire e il fare, val piu la pratica, ecc. In certi casi tali scorciature si trasformano in modi di dire proverbiali che vengono impiegati nella sintassi della frase come un qualsiasi altro elemento del discorso. Talora, come nel nostro caso, conservando un'eco o qualcosa del senso del proverbio: acqua passata, mori fanciullo, ponti d'oro, scherzo da villano, saltar dalla finestra, ecc. Talaltra assumendo maggior autonomia, tanto che il legame col proverbio si indebolisce e non si avverte quasi più:acqua a catinelle, mari e monti, un po' per uno, ecc.

È interessante notare che i primi esempi del nostro modo di dire compaiono intorno alla metà dell'Ottocento, forse per influenza dei Promessi sposi, ai quali si deve comunque la notorietà del proverbio. E, almeno nei primi tempi e fino al nuovo secolo, sempre nella forma col senno del poi: "se egli è cosa facile il dar consigli col senno del poi, a mille e più anni di distanza, non è meno temeraria ed insulsa" (“Civiltà cattolica”, XI, vol. viii, I860, p. 298).

Dobbiamo quindi chiederci perché dal proverbio, che fino allora presentava la forma del senno di poi, sia scaturita una locuzione nella quale quell'avverbio vien trasformato in sostantivo. Scartate le motivazioni formali (assimilazione alla preposizione articolata in prima posizione) che avrebbero potuto valere anche per il proverbio, si tratta di qualcosa di piu sottile e profondo. Obliterando la seconda parte del proverbio con il suo riferimento alla morte e al giudizio che attende i credenti, era necessario consolidare il modo di dire in una più chiara espressione nominale, nella quale il poi 
sostantivo indicasse, se non l'aldilà, almeno un qualche tempo venturo; mentre l'avverbio, in mancanza di un verbo, rendeva l'espressione fraseologica generica e monca. Insomma, sostantivando l'avverbio in certo modo s'inglobava nella locuzione ciò cui alludeva la seconda parte del proverbio: "il tempo del poi" - fosse quello della vita dopo la morte o di un'epoca storica successiva - che consente quel giudizio più veritiero e schietto di quando si accetta la prospettiva e l'etica propria della religione; invece "il senno di poi" non è altro che quell'usuale senno a posteriori che avevano gli antichi e che si ha sempre ogni volta che ci si volga indietro a cose fatte: un giudizio "a scoppio ritardato" che non va al di là dei nostri limiti umani.

La diffusione a metà Ottocento del modo di dire col senno del poi propagginò la variante sostantivata anche al proverbio, a partire dall'esempio di Guerrazzi che si è citato. Mentre i vocabolaristi, non solo continuarono a registrare il proverbio nella forma tradizionale attestata dagli scrittori fino al Manzoni, ma vi conformarono anche il modo di dire, riportandolo di solito nella forma col senno di poi. Si deve probabilmente proprio a tale indicazione lessicografica (a meno di non imputare la cosa alla crescente secolarizzazione della società italiana) l'attuale altalena fra le due varianti cui si è accennato all'inizio: non è che lo si faccia sempre, ma chi è incerto e segue ciò che dicono i vocabolari, finisce per creare ulteriore incertezza. Ho pero l'impressione che anche quando, per rispetto della "norma" vocabolaristica generatrice di tale aporia, i parlanti ripiegano sul senno di poi, quel poi, pur senza articolo, sia sentito più come un sostantivo che come un avverbio.

A questo punto ognuno potrà trarre le sue conclusioni. Oscillazioni, dovute ai più diversi fattori, sono sempre presenti nelle vicende delle lingue e dunque anche nei proverbi e nei modi di dire. L'epoca attuale, esaltando l'esattezza delle scienze e lo standard delle tecniche, pretenderebbe di normalizzare omogenizzare uniformare anche la lingua: e non di rado ci riesce. Ma la lingua non è fatta di assiomi e di logica: vive negli intrichi e negli ingorghi delle società umane, respira e palpita con la mente e il cuore dei singoli individui: non può farne a meno, se vuol andare avanti, se si vuol andare avanti. Respiri e palpiti che sono quelli del nostro povero cuore e dei nostri pensieri debolissimi, sempre incerti, timorosi, confusi, contraddittori, almeno fino al giorno del giudizio in cui finalmente vedremo ogni cosa in modo più chiaro: "col senno del poi". Così, per ora, in questa vita terrena, non dovremmo far altro che usare la lingua che ci è data, affidandoci alla nostra imperfetta competenza, che comunque è sempre migliore e più sicura di quella dei periti, degli scienziati, dei sofisti, capaci di molte cose, anche di sezionare e notomizzare alla perfezione ciò che non andrebbe toccato. Come la lingua che è vita e va vissuta in modo vero e serio, e soprattutto con spirito libero e coraggioso.

\section{Appendice}

Devo alla cortesia di Paolo Rondinelli, curatore dell'edizione critica e commentata della grande raccolta di proverbi di Francesco Serdonati (Lamole in Chianti?, I540-Roma, I6I6?), edizione la cui prima parte è di prossima pubblicazione presso l'Accademia della Crusca, l'inedito brano che segue, tratto dalla lettera D:

550. Del senno di poi ne son piene le fosse. Quel che dicono i latini: Sero sapiunt. Petrarca, cap. 3 d'Amore: / «E come tardi dopo 'l danno intendo». Altri dicono / Il pentirsi da sezzo nulla giova. Giovanni Villani, 1. 7, I5: «E venuto in Prato hebbono molti ripitii, ma dopo cosa male consigliata e peggio fatta invano è il pentir poi». E Matteo Villani, 1. 3, C. 32: «Ma, dopo il fatto, aggiunta di vituperio è il pentire, che la soma sotto il tirannesco giogo convenne loro portare». E 1. 4, C. 32: / «Ma la guerra è di questa natura, che commesso il fallo seguita la penitentia senza rimedio le più volte». Leone imperadore, comandamento 20, num. 36: «Perciò che, dopo l'haver danno ricevuto, questa tarda penitenzia non 
apporterà niun giovamento». Boccaccio, 1. 4 del Filocolo, 373: «Gli avvedimenti di dietro poco vagliono, o niente». Altri dicono / Il pentirsi dopo 'l fatto nulla vale. Pietro Nelli [Rime piacevoli], 9r: / «Ma, se valesse il pentirsi da poi, / Pochi si troverian che la seconda / Volta non fesser meglio i fatti suoi».

Si può notare che Serdonati accosta il nostro proverbio, oltre che all'espressione latina Sero sapiunt, a due proverbi simili (Il pentirsi da sezzo nulla giova e Il pentirsi dopo il fatto nulla vale), aggiungendo diversi esempi di scrittori che li impiegano in modo vario. Il curatore del testo, che nella sua edizione ricostruirà l'intero reticolo delle fonti, mi scrive a proposito del latino Sero sapiunt, di cui si era già occupato nel Liber proverbiorum di Lorenzo Lippi (ed. critica a cura di Paolo Rondinelli, Bologna, Bononia University Press, 20II):

Lorenzo Lippi (Colle Val d'Elsa, I446?-Pisa, I485), fra i proverbi che condivide con gli Adagia (I508) di Erasmo, ha anche Cumani non sentiunt (mentre Erasmo, Adagia, I.5.6r ha Cumani sero sapiunt). Esiste anche la variante Sero sapiunt Phryges, che prende di mira i Troiani, rei di aver iniziato a pensare alla restituzione di Elena a Menelao solo in seguito a perdite enormi (cfr. Tosi, Diz. delle sentenze latine e greche cit., ${ }^{\circ}$ II64: Troppo tardi rinsaviscono i Frigi). Anche questo si trova negli Adagia di Erasmo (I.r.28). Sono "proverbia a gentibus", o proverbi-blasoni, che traggono spunto da caratteristiche negative di popoli o terre del mondo antico. La fonte del Lippi è la Geographia di Strabone (XIII 3, 6) latinizzata da Guarino Veronese e da Gregorio Tifernate per papa Niccolò V, forse letta nell'incunabolo stampato a Venezia da Vindelino da Spira nel I472. Dietro a Cumani non sentiunt si cela il seguente aneddoto riportato da Lippi (pp. 435-36): «I. Carpuntur Cumani ut homines insulsi et hebetes ob hanc causam, ut quidam putant quod CCC annis post eius urbis aedificationem portus vectigalia exegerunt, qum antea populus eiusmodi proventus non haberet. 2. Quare obtinuit opinio, quod hi homines sero sentirent, cum urbem ad mare habitarent. Sunt qui aliter sentiant, quod publica pecunia mutuo accepta porticus construxerunt. 3. Postea, cum pecuniam constituta die non reddidissent, a deambulatione arcebantur. 4. Sed, cum pluviae essent, in dedecus quoddam creditores edictum mittebant, ut Cumani porticus subirent, et cum praeco inclamaret: «Porticus subite!», dictum est proverbium, quod Cumani non sentiant, quod in pluvia porticus subeunda sit, etiam si nemo per praeconem admoneat».

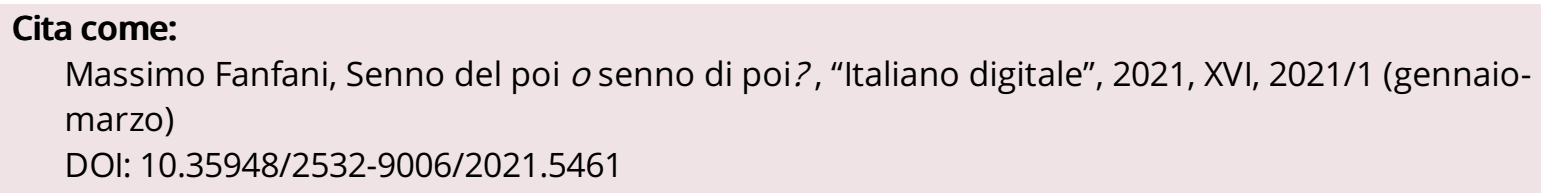

